\title{
Bilateral Olecranon Fracture and Radio-Humeral Dislocation
}

Fred Kalande

School of Medicine, Egerton University

Correspondence to: Dr. Fred Kalande, P.O. Box 20115, Egerton, Kenya. Email: kalandefred@yahoo.com

\section{Summary}

Olecranon fractures constitute $10 \%$ of traumatic fractures in the upper limb however; bilateral traumatic olecranon fracture is rare. They follow a fall on a flexed elbow or strike on the point of the elbow or in patients with other systemic pathology such as Rheumatoid arthritis or sarcoidosis. Bilateral fractures with posterior radio humeral dislocation are even rarer and offer a management dilemma. We report one case of traumatic bilateral olecranon fractures with posterior radiohumeral dislocation without any concomitant systemic disease. After imaging studies, the olecranon fracture was classified using Mayo classification and the patient was prepared for surgery under general anesthesia. Tension band wiring fixation was performed on the left oblique olecranon fracture and contoured reconstruction

\section{Introduction}

Trauma to the upper limb is devastating as it affects the livelihood of the patient and injuries around the elbow are associated with posttraumatic loss of joint range of motion. Unilateral olecranon fractures constitute $10 \%$ of upper limb fractures (1). They follow trauma either as a fall from a height on the elbow or motor vehicle accident injury as a side swipe. Bilateral fractures of olecranon are very rare. They have been seen in patients with other systematic pathology such as rheumatoid arthritis or sarcoidosis $(2,3)$. Bilateral traumatic olecranon fractures in healthy individual are quite rare. This paper highlights this rare injury and the challenges in treatment and outcome.

\section{Case Report}

A 30yr old carpenter on outstretched hands fell from a height of about 3 meters while repairing a roof. He sustained injuries to both elbows, and bruises over the trunk; there were no other associated injuries. There plate fixation was performed on the right comminuted fracture. The recovery was uneventful and through intense physiotherapy the elbow function was quite impressive. The patient was able to perform daily living activities on his own having achieved Mayo elbow score (MES) of 90 on the right and 85 on the left.

Key words: Bilateral Olecranon fracture, RadioHumeral dislocation, Mayo classification

Ann Afr Surg. 2018;15(2):77-80

DOI:http://dx.doi.org/10.4314/aas.v15i2.10

(C) 2018 Author. This work is licensed under the

Creative Commons Attribution 4.0 International

License.

Conflicts of Interest: None

Funding: None

was no loss of consciousness during and after the fall. On examination, he had grossly swollen tender elbows with minimal bruising over the posterior surface.

No bleeding or cuts were seen.

Brachial and radial pulses were present on both limbs. Sensation was normal. The elbows were splinted flexed at $90^{\circ}$ and intramuscular analgesia administered. A radiograph showed bilateral fractures of the olecranon with posterior radio-humeral dislocation (Figure 1).

Informed consent was obtained from the patient for open reduction and internal fixation to be performed under general anaesthesia. Intraoperatively, prophylaxis antibiotic (2grams cefuroxime) was given, tourniquet was applied and in supine position; bilateral posterior elbow incisions were made to expose the fractures; which were then reduced and prefixed with $\mathrm{K}$ wires. The left olecranon fracture was finally fixed by tension band wiring due to its transverse nature while the right was reduced and fixed with a contoured reconstruction plate and screws because it was comminuted. 
The dislocations reduced spontaneously after the fracture fixation. The annular ligaments were repaired using absorbable $2 / 0$ polyglactin suture. Stability intra operatively was ascertained by going through a full range of motion without any loss of reduction.

The elbows were then immobilized in back slab at $90^{\circ}$ of flexion after the surgery. The post reduction radiographs were satisfactory (Figure 2).

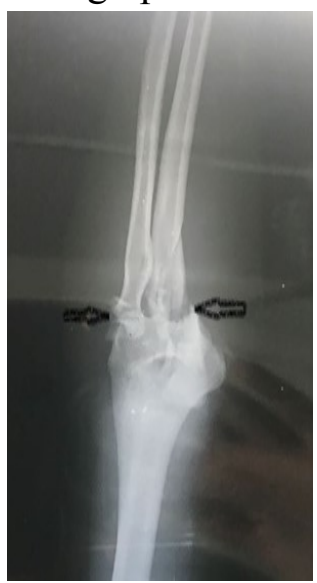

Figure 1: Preoperative radiograph of right elbow (Fracture demonstrated by the arrows)

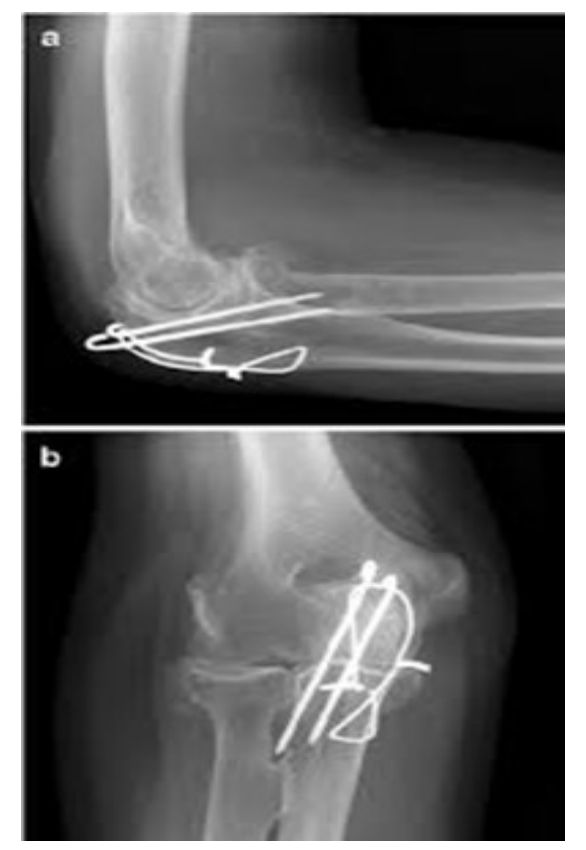

Figure 2: Postoperative radiograph of the right elbow

After 7 days, active and passive range of motion was commenced with the help of a physiotherapist. Oral Indomethacin $25 \mathrm{mg}$ thrice daily was given for two weeks to prevent heterotrophic ossification. The patient recovered well and the wounds healed uneventfully. Elbow stability was satisfactory, achieving range of motion (ROM) of $0-120^{\circ}$ on the left and $0-110^{\circ}$ on the right with pronation and supination of $0 \quad-20^{0}$ achieved on both elbows. Functional outcome was done using Mayo Elbow Score (MES) with a score of 90 on the right (excellent) and 85 on the left (excellent) (Table 1) .After six months follow up, the patient was able to return to normal occupation but with mild discomfort on both elbows due to hardware friction under the skin.

Table 1: Mayo Elbow Score (MES)

\begin{tabular}{|l|l|l|}
\hline Function & Definition & Points \\
\hline Pain & None & 45 \\
\cline { 2 - 3 } & Mild & 30 \\
\cline { 2 - 3 } & Moderate & 15 \\
\cline { 2 - 3 } & Severe & 0 \\
\hline Stability & Arc $>100^{0}$ & 20 \\
\cline { 2 - 3 } & Arc $50-100^{0}$ & 15 \\
\cline { 2 - 3 } & Arc $<50^{0}$ & 5 \\
\hline \multirow{5}{*}{ Function } & Stable & 10 \\
\cline { 2 - 3 } & Moderate instability & 5 \\
\cline { 2 - 3 } & Severe instability & 0 \\
\cline { 2 - 3 } & Comb hair & 5 \\
\cline { 2 - 3 } & Feed & 5 \\
\cline { 2 - 3 } & Hygiene & 5 \\
\cline { 2 - 3 } & Wear shirt & 5 \\
\cline { 2 - 3 } & Wear shoes & 5 \\
\hline
\end{tabular}

Total score 100; Excellent result $>90$, Good result 75-89, Fair 60 74 , Poor result $<60$

\section{Discussion}

Olecranon fractures are commonly unilateral with bilateral fractures being quite rare (5). Bilateral olecranon fractures with elbow dislocation are rarer. They commonly follow a fall on the elbow or a blow on the tip of the elbow. A significant number occur in patients with concomitant systemic pathology such as rheumatoid arthritis; sarcoidosis or osteogenesis imperfecta $(6,7,8$,). Citlak et al described two cases with bilateral olecranon fractures after a fall, however they were not associated with radio humeral dislocation. (11)We describe bilateral olecranon and posterior radio-humeral dislocation after a fall in an adult with no systemic disease, a variant of an earlier described injury; the terrible triad which defined as fracture coronoid; fracture head of radius and elbow dislocation $(9,10)$. Various classifications are used for 
olecranon fracture but Mayo classification is the most commonly used. It is based on stability, displacement and comminution of the fracture. It was applied in our case and formed a guide of whether to plate as was done on the right or tension band wiring (TBW) as was done on the left (4).Our patient had Mayo IIIA on the left where the olecranon fracture was are displaced, associated joint dislocation and IIIB on the right due to the concomitant comminution (13). There are few cases of bilateral Mayo IIB managed both by $\operatorname{TBW}(4,11)$.

With timely intervention the patient gained well near normal elbow function. Mayo elbow score is a valuable monitoring tool for improvement of function following elbow surgery. Amit Patel and Vullasetal (6) had two cases which had fracture and dislocation and the results were similar as in our case. The treatment technique followed in our case was as that for unilateral fracture using Mayo classification (8). John D; Miller and Ford $(5,9)$ described plates for comminuted fractures and TBW for linear fractures. Hak and Colliday (4) did describe the treatment option for fixing different types of olecranon fractures. Bilaterality influenced few aspects of the management except the duration of surgery and type of anesthesia; brachial plexus block in bilateral fractures is associated with lignocaine toxicity and was not used in our case (10). This would impact on patients with comorbid conditions that preclude use of general anesthesia (9). In our case rehabilitation was a more arduous task for the patient who was however able to achieve good early range of motion with the help of physiotherapist, we achieved; Mayo Elbow Score (MES) of 90 on the right and 85 on the left. Indomethacin was given to prevent heterotrophic ossification as advocated by others (12 13, 14). Douglas and Cannada had shown in their study that indomethacin did prevent extra- osseous ossification our case had similar outcome and we recommend in such cases (13). Elbow instability following such complex fracture dislocation with associated ligament tear require ligament repair for good function. On follow up at 6 months both elbows were stable. We recommend its repair at reduction to achieve good and stable elbow function.

\section{Conclusion}

Management of bilateral olecranon fracture is feasible in one single operation. The fixation, approach and hardware used is still guided by the pattern of the fractures and the quality of bone.Active and passive physiotherapy, together with indomethacin are initiated early to avoid debilitating elbow stiffness that would incapacitate the victim.

\section{References}

1. Wiegand L, Bernestein J, Ahm A. Fractures in Brief: Olecranon Fractures. Clin Orthop Relat Res.2012; 470:3637 -41.

2. Kirman S, DraviajahK, Madegowda B etal Spontaneous Bilateral Olecranon Fracfures in Rheumatoid Patient. Ann R Coll Surg Engl. 2008;90: 1-3.

3. O Daily BJ, Harty JA, O’Malley N et al. Bilateral Olecranon Fracture as First Presentation of Sarcoidosis. Case Report and Review of Literature. J Shoulder Elbow Surg. 2008;17: 1-5.

4. Hak DJ, Golladay CJ. Olecranon Fracture: Treatment Options. J Am Acad Orthop Surg. 2008; 8(4); $266-75$.

5. John J, Miller D, Ford S, et al Olecranon Fractures Tension Band Wire or Plate.

Orthopaedic proceedings British education society 2018; 9: 251 -7.

6. Patel A, UllasT, Vikas et al. Simultaneous Bilateral Traumatic Olecranon Fracture: A Rare Case Report. IOSR journal of dental and medical sciences 2016; 15(4): 65 -76.

7. Guoging Z H A, Xiaofeng N J U, Weiguang Y U. Severe injury of Bilateral elbow joints with unilateral terrible triad injury of elbow. International journal of clinical and experimental medical science 2015; 8(8):14212-14220.

8. Schweitzer G. Bilateral Avulsion Fracture of Olecranon Apophyses. Arch Orthop Trauma Surg. 1988; 107: 181 -9

9. Giannicola G, Manauzzi E, Cinotti G. Management of Bilateral Complex Elbow Fractures and Proximal Radio - ulna Dislocation: A Case Report. Musculoskeletal Surg. 2012; 96 (Suppl 1): s87-s92. 
10. Sish,T, IIB Bulin. Techniques of Fracture Fixation, Online journal management system 2012; 1:34 -7.

11. Citlak A, Guselb N, Kerinoglue V et al Bilateral isolated olecranon fracture after trauma report of 2 cases. Journal of experimental and clinical medical science 2014; 31:107 -10.

12. O'Driscoll SW, Jupiter JB King, CG et al Unstable elbow. J Bone Joint Surg Br. 2000; 82: 724 -5.

13. Cabanela. Morrey. The elbow and its disorders. $3 \mathrm{~d}$. Philadelphia, Saunders; 2000. pp. 365-379.

14. Kenneth J Koval, Joseph D Zuckerman, hand book of fractures. Third edition 2010 Lippincott Williams and Wilkins chapter 19 olecranon 2010 Page204 -205. 